\title{
Médecins militaires: perfectionnement et formation postgraduée une occasion de chance
}

Gianpiero A. Lupia, Adrian Leutenegger ${ }^{b}$

a Dr méd., Divisionnaire, chef des Affaires sanitaires/ médecin en chef de l'armée

b Prof. Dr méd., doyen de l'Académie suisse de médecine militaire et de catastrophe (ASMC)
Correspondance:

Peter Kehl

BLA - Affaires sanitaires Méd mil enseignement et recherche, ASMC Worblentalstrasse 36

$\mathrm{CH}-3063$ Ittigen
Au cours des dernières années le service sanitaire de l'armée a connu des changements fondamentaux. Les époques des hôpitaux militaires improvisés sous tentes, dans des abris souterrains et des bâtiments scolaires font définitivement partie du passé.

Toutefois la tâche du service sanitaire reste comme auparavant de préserver la force de combat et le moral de la troupe dans toutes situations et, si possible, de les rétablir lorsqu'ils peuvent être affectés par des problèmes de santé. Le profil d'exigence du service sanitaire se situe actuellement à l'échelle de la santé publique et tous les militaires ont droit aujourd'hui durant leur service à une prestation médicale équivalente à celle de la vie civile. Des éléments du service sanitaire de l'armée peuvent également être engagés sur le plan civil dans des situations extraordinaires au profit de la santé publique.

Le projet de l'Académie suisse de médecine militaire et de catastrophe (ASMC) coordonne le perfectionnement et la formation postgraduée médico-militaires sur l'ordre du Conseil fédéral. Actuellement une formation spécialisée de qualité est offerte en particulier aux jeunes collègues en formation (études, postgraduée et continue) en étroite collaboration entre l'armée et nos facultés de médecine avec l'avantage d'être reconnue comme études et formation postgraduée spécialisée. Les études et le service militaire sont compatibles entre eux et les cours de répétition peuvent être uniquement effectués en tant que médecins formés.

Les médecins militaires diplômés ont la possibilité dans le cadre de leurs obligations de service d'accéder gratuitement à de nombreux cours de formation postgraduée lesquels sont également exigés en partie dans la formation médicale de certaines spécialités. En outre, trois mois de formation médicale peuvent être comptabilisés comme service militaire et les postes correspondants de formation sont financièrement soutenus pendant cette période par un dédommagement compensatoire (modèle 9+3).

De nombreuses et diverses possibilités d'engagement sont ouvertes pour les médecins militaires formés: service de médecin de troupe lors des obligations de service dans les écoles et les cours de répétition, engagements dans un des sept centres de recrutement ou des six centres médico-régionaux, unités de sauvetage avec le Swiss Medical Unit ou aussi des engagements à l'étranger sur une base volontaire avec la Swisscoy KFOR ou dans le cadre de missions humanitaires du CSA.

La santé publique profite de la formation de qualité et des expériences professionnelles supplémentaires de nos médecins militaires dans la planification et la garantie de la prise en charge médicale de notre population lors de catastrophes, d'épidémies et d'événements majeurs.

Aujourd'hui la formation est attrayante pour des médecins militaires et correspond à une véritable occasion de chance. Des indications détaillées concernant le profil d'exigence, l'accès à la formation, les dédommagements financiers, les obligations de service ultérieures ainsi que l'ASMC peuvent être consultées sur le site www.vbs-ddps.ch/internet/groupgst/de/home/ sanit/neu.html ou au moyen du système de recherche Google sous ASMC. 


\section{An Militärärzte (Offiziere)}

Die Sanität in der Logistikbasis der Armee offeriert Ihnen im Rahmen des Projektes Schweizerische Akademie für Militär- und Katastrophenmedizin (SAMK) die folgenden Weiter- oder Fortbildungskurse, die zum grossen Teil schon durch die FMH anerkannt sind und die auch an Ihre jährliche Militärdienstpflicht angerechnet werden.

Interessenten wollen sich bitte rechtzeitig anmelden: www.vbs-ddps.ch/internet/groupgst/de/ home/sanit/neu/neu2.html

\section{Aux médecins militaires (officiers)}

L'élément sanitaire de la base logistique de l'armée vous offre, dans le cadre du projet Académie suisse de médecine militaire et de catastrophe (ASMC), les cours suivants de formation postgraduée ou continue, déjà en grande partie reconnus par la FMH et imputables à votre service militaire annuel obligatoire.

Les intéressés voudront bien s'annoncer à temps: www.vbs-ddps.ch/internet/groupgst/de/ home/sanit/neu/neu2.html

\begin{tabular}{|c|c|c|}
\hline \multicolumn{3}{|l|}{$\begin{array}{l}\text { Kursangebote der SAMK / Offres des cours ASMC } \\
*=\text { ausgebucht/occupés }\end{array}$} \\
\hline \multicolumn{3}{|l|}{ Anästhesie und Reanimation in Basel } \\
\hline \multicolumn{3}{|l|}{ Kurse in deutscher Sprache. } \\
\hline Anästhesiekongress (englisch) & 16./17.3.2007 & BS 1 \\
\hline * Notarztkurs (Grundlagen der präklinischen Notfallmedizin) & $22 . / 23.3 .+29 . / 30.3 .2007$ & BS 2 \\
\hline Notarztkurs (Grundlagen der präklinischen Notfallmedizin) & 15./16.11.+ 22./23.11.2007 & BS 3 \\
\hline${ }^{*}$ ACLS (Advanced Cardiovascular Life Support) & 26./27.4.2007 & BS 4 \\
\hline * ACLS (Advanced Cardiovascular Life Support) & 18./19.10.2007 & BS 5 \\
\hline${ }^{*}$ ATLS (Advanced Cardiovascular Life Support) & 27.-29.8.2007 & BS 6 \\
\hline * PALS (Pediatric Advanced Life Support) & $30 . / 31.8 .2007$ & BS 7 \\
\hline TPAM (Training in Prehospital Airway Management) & 19.11.2007 & BS 9 \\
\hline * ACMS (Advanced Complication Management) & 27.3.2007 & BS 10 \\
\hline ACMS (Advanced Complication Management) & 28.3.2007 & BS 11 \\
\hline \multicolumn{3}{|c|}{ Médecine et management en cas de catastrophe et de guerre à Lausanne } \\
\hline \multicolumn{3}{|l|}{ Cours en langue française ou allemande. } \\
\hline $\begin{array}{l}\text { Catastrophe à effets limités (accidents majeurs); cours pour } \\
\text { professionnels de la chaîne des urgences. Formation pré- } \\
\text { requise: VD 1[M1] (sauf pour médecins d'urgence SSMUS) }\end{array}$ & 26.-28.4.2007 & VD 2 [M2] \\
\hline $\begin{array}{l}\text { Catastrophe à effets limités (accidents majeurs); cours de } \\
\text { conduite pour médecins-chefs des secours et ambulanciers } \\
\text { chefs de secours. Formation pré-requise: VD } 2 \text { [M2] }\end{array}$ & 14./15.6.2007 & VD 3 [M3] \\
\hline $\begin{array}{l}\text { Catastrophes majeures dans les pays industrialisés. } \\
\text { Formation pré-requise: VD } 3 \text { [M3] }\end{array}$ & 13./14.9.2007 & VD 4 [M4] \\
\hline $\begin{array}{l}\text { Catastrophes humanitaires complexes. Formation pré- } \\
\text { requise: VD 1[M1] }\end{array}$ & 24.-26.10.2007 & VD 5 [M5] \\
\hline $\begin{array}{l}\text { Catastrophes: planification hospitalière. Formation pré- } \\
\text { requise: VD } 1 \text { [M1] }\end{array}$ & $6 . / 7.12 .2007$ & VD $6[\mathrm{M} 6]$ \\
\hline ACLS & $30 . / 31.10 .2007$ & VD 7 \\
\hline Cours de médecine d'urgence (SSMUS) & 18.-21.9.2007 & VD 8 \\
\hline PALS & 19./20.11.2007 & VD 9 \\
\hline $\begin{array}{l}\text { * Sanitätsdienstl. Führung Grossereignis (SFG) (Teil A: } \\
\text { «Knowledge», Grundlagen, Prinzipien, Partner). } \\
\text { Voraussetzungen: Notarzt SGNOR oder Rettungssanitäter } \\
\text { in Kaderposition (oder äquivalent) }\end{array}$ & 2.-4.5.2007 & VD A [SFG-A] \\
\hline $\begin{array}{l}\text { Sanitätsdienstl. Führung Grossereignis (SFG) (Teil B: } \\
\text { «Training», Kommunikation und Teaminteraktion). } \\
\text { Voraussetzung: VD A [SFG-A] }\end{array}$ & 15./16.11.2007 & VD B [SFG-B] \\
\hline
\end{tabular}




\begin{tabular}{|c|c|c|}
\hline \multicolumn{2}{|l|}{ Kursangebote der SAMK / Offres des cours ASMC } & \\
\hline \multicolumn{3}{|l|}{ Chirurgie à Genève } \\
\hline \multicolumn{3}{|l|}{ Cours en langue française. } \\
\hline Cours de chirurgie de guerre et de catastrophe (théorie) & 16./17.10.2007 & GE 1 \\
\hline $\begin{array}{l}\text { Cours de chirurgie de guerre et de catastrophe (pratique). } \\
\text { Formation pré-requise: GE } 1\end{array}$ & 18./19.10.2007 & GE 2 \\
\hline${ }^{*}$ ATLS & 10.-12.5.2007 & GE 3 \\
\hline${ }^{*}$ ATLS & $30.8-1.9 .2007$ & GE 4 \\
\hline \multicolumn{3}{|l|}{ Innere Medizin, Infektiologie in Bern } \\
\hline \multicolumn{3}{|l|}{ Kurse in deutscher Sprache. } \\
\hline 5. Systematisches Infektiologie-Curriculum & 31.10 .2007 & BE $1 \mathrm{~A}$ \\
\hline 10. Berner Infektiologie-Symposium & 1.11.2007 & BE 1B \\
\hline $\begin{array}{l}\text { 2. Systematisches Curriculum Internmedizinische Notfall- } \\
\text { medizin }\end{array}$ & 21.9.2007 & BE $2 \mathrm{~A}$ \\
\hline 6. Berner Notfall-Symposium & 20.9.2007 & $\mathrm{BE} 2 \mathrm{~B}$ \\
\hline${ }^{*}$ ACLS-Kurs 10 (Bern) & 8./9.5.2007 & BE 3 \\
\hline${ }^{*}$ ACLS-Kurs 19 (St. Gallen) & 25./26.10.2007 & BE 4 \\
\hline * ACLS-Kurs 23 (Zürich) & 15./16.11.2007 & BE 5 \\
\hline Dienstarztkurs SGNOR & 7./8.6.+21./22.6.2007 & BE 6 \\
\hline \multicolumn{3}{|l|}{ Katastrophen- und Wehrpsychiatrie in Zürich } \\
\hline \multicolumn{3}{|l|}{ Kurse in deutscher Sprache. } \\
\hline $\begin{array}{l}\text { Resilience, Stressverarbeitung und Burnoutprophylaxe für } \\
\text { (leitende) Notärzte in Kooperation mit den leitenden Not- } \\
\text { ärzten des Universitätsspitals Zürich. Zulassungsbedingung: } \\
\text { ATLS-Kurs }\end{array}$ & 30.3., 27.4. und 1.6.2007 & $\mathrm{ZH} 1$ \\
\hline $\begin{array}{l}\text { Psychologische Nothilfe (PNH) in der Armee: eine Manage- } \\
\text { mentaufgabe (Workshop) }\end{array}$ & 18./19.10.2007 & $\mathrm{ZH} 2$ \\
\hline${ }^{*}$ ATLS & 4./5.6.2007 & $\mathrm{ZH} 4$ \\
\hline${ }^{*}$ ATLS & $31.5 / 1.6 .2007$ & ZH 5 \\
\hline${ }^{*}$ ATLS & 6./7.9.2007 & ZH 6 \\
\hline * PALS & 12./13.7.2007 & $\mathrm{ZH} 7$ \\
\hline * PHTLS & 17./18.9.2007 & $\mathrm{ZH} 8$ \\
\hline \multicolumn{3}{|l|}{ Centre spécialisé pour soins, Lausanne } \\
\hline \multicolumn{3}{|l|}{ Cours en langue française. } \\
\hline Soins physiques & 21.-23.3.2007 & SM 2 \\
\hline Approches interculturelles/risques/sécurité/ressources & 20.-22.9.2007 & SM 3 \\
\hline Aspects psychologiques & 24. -26.10 .2007 & SM 4 \\
\hline L'expatrié dans sa mission & 6. -8.12 .2007 & SM 5 \\
\hline \multicolumn{3}{|l|}{ Fachzentrum für Transfusionswesen } \\
\hline \multicolumn{3}{|l|}{ Kurssprachen: Deutsch, Französisch, Italienisch, Englisch. } \\
\hline 14. Schweizerisches Notfallsymposium & 13./14.4.2007 & TW 1 \\
\hline
\end{tabular}

